which can be related to $\mathrm{L}(+)$ tartaric acid are correct, and (I) represents the absolute stereochemical structure of morphine.

Department of Chemistry,

I. R. C. Bick

University of Tasmania,

Hobart.

Oct. 18.

${ }^{1}$ Schöpf, C., $A n n ., 452,211$ (1927). Schöpf, C., and Pfeifer, T., $A n n$., 483, 157 (1930).

Fieser, L. F., and Fieser, M., "Natural Products Related to Phenanthrene", 24 (3rd edit., Reinhold, New York, 1949).

${ }^{3}$ Holmes, H. L. (unpublished).

4 Rapoport, H., and Payne, G. B., J. Org. Chem., 15, 1093 (1950). Rapoport, H., and Payne, G. B., J. Amer. Chem. Soc. (in the press).

' Clough, G. W., J. Chem. Soc., 113, 526 (1918).

${ }^{\circ}$ Leithe, W., Ber., 63, 1498 (1930).

' Leithe, W., Ber., 63, 2343 (1930); 64, 2827 (1931) ; 67, 1261 (1934).

${ }^{8}$ Bijvoet, J. M., Peerdeman, A. F., and van Bommel, A. J., Nature, 168, 271 (1951).

\section{S-Methyl Dithizone and the Structure of Metal Dithizonates}

ALthovGH diphenylthiocarbazone ('dithizone', I or II, $X=\mathrm{H}$ ) is probably the most widely used of all organic reagents for trace-metal determinations, its structure, and more particularly the structure of its metal complexes, are still in doubt. Several metals form two dithizone complexes, that with the lower proportion of metal being termed the keto-form, the metal-rich complex being known as the enol form. Following Hellmut Fischer ${ }^{1}$, it is commonly supposed that in the keto form it is a hydrogen atom from the imido group of dithizone that is replaced by a metal (I, $X=$ monovalent metal ; IV, $Y=$ divalent metal) ; whereas in the enol form the hydrogen atom from the sulphydryl group of the tautomeric form of the reagent (II, $X=\mathbf{H}$ ) is replaced by metal as well (II, $X=$ monovalent metal ; V, $Y=$ divalent metal). There is virtually no experimental evidence for these formulations, which are nevertheless reproduced in standard text-books ${ }^{2}$, though some of them are impossible on steric grounds alone.<smiles>S=C1[Y]=CN[W]=N1</smiles>

(I)

(IV)<smiles>S=c1nn[nH][pH][nH][nH]1</smiles><smiles>[X]c1nnnnn1</smiles>

(II)<smiles>c1nnnnn1</smiles>

(III)

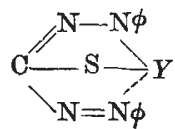

(V)

Treatment of a chloroform solution of 'keto' silver dithizonate with methyl iodide has now been found to give a monomethyl derivative of dithizone as an almost black solid with a bronze reflex, melting point $118-120^{\circ}$. Now, since it is a monobasic acid ${ }^{3,4}$, dithizone dissolves in aqueous alkali, and the action of dimethyl sulphate upon such a solution gives the same methyl derivative. That the methyl group is attached to sulphur and not to nitrogen is proved by the synthesis of the same methyl-dithizone by the action of sodium methyl mercaptide upon an alcoholic solution of $\omega$-chlorodiphenylformazan (III, $Z=\mathrm{Cl}$ ), itself prepared by coupling chloromalonic acid with benzenediazonium chloride in acid solution. In each

case the $\omega$-methylmercaptodiphenylformazan (III, $Z=\mathrm{CH}_{3} . \mathrm{S}-$ ) was purified chromatographically on activated alumina, and the identity of the substances prepared by the three different routes was established by analyses, mixed melting points and by their giving identical absorption spectra in carbon tetrachloride. It is noteworthy that, when a solution in an organic solvent is exposed to sunlight, the absorption spectrum changes in a manner similar to that previously observed in the case of other $\omega$-substituted formazans $^{5}$.

As expected from its structure, $\omega$-methylmercaptodiphenylformazan is insoluble in aqueous alkalis and does not give rise to metal complexes under the conditions where metal dithizonates are readily formed. Thus, it must be the thiol group which confers acidic properties upon dithizone $e^{3,4}$, and it appears probable that it is the hydrogen of this thiol group which is replaced by a metal atom in the formation of a metal 'keto' dithizonate. Further experiments are now in progress in order to elucidate the structure of the enol complexes and towards the synthesis of an N-methyl dithizone (I, $\mathrm{X}=\mathrm{CH}_{3}$ ), which in its thiol form should show acidic properties and be able to form metal complexes, although the internal hydrogen-bonding which probably occurs in dithizone and other derivatives of diphenylformazan ${ }^{3,5}$ would here be inhibited.

Treatment of $\omega$-chlorodiphenylformazan (III, $Z=$ Cl) with sodium hydrosulphide yields dithizone itself and affords a convenient synthesis of this reagent labelled with radiosulphur.

H. Irving

C. F. BELL

Inorganic Chemistry Laboratory, University of Oxford. Nov. 26.

1 Fischer, Hiss, Veröffentlich. Siemens-Konzern, 4, 158 (1925); Angev. Chem., 47, 685 (1934).

' Welcher, F. J.. "Organic Analytical Reagents"', 3, 468 (Van Nostrand, 1949) Milton, R. F and Waters, W. A." "Methods of Quantitative 1949). Milton, R. F., and Waters, W. A., "Methods

- Irving, H., Cooke, S. J. H., Woodger, S. C., and Williams, R. J. P., J. Chem. Soc, 1847 (1949).

- Irving, H., and Bell, C. F., J. Chem. Soc. (in the press).

s Hausser. I., Jochel, D., and Kuhn, R., Ber., 82, 515 (1949).

\section{Organic Derivatives of Montmorillonite}

THE supposed analogy between hydrogen-saturated montmorillonite and a weak organic acid has encouraged several investigators, notably Berger ${ }^{1}$ and Deuel ${ }^{2-4}$, to attempt to esterify the montmorillonite acid using standard organo-chemical methods.

In our opinion, the experimental evidence so far presented to establish the course and extent of the esterification reaction is inconclusive. Thus, Berger ${ }^{1}$ determined the methoxyl content of the product obtained by treating hydrogen montmorillonite with diazomethane in ether without showing that it was free of methyl alcohol and ether. Some of the experiments of Deuel are open to similar criticism. Deuel ${ }^{2-4}$ generally determined the extent of esterification by the decrease in the exchange capacity of montmorillonite. We have found that exchange-capacity measurements made by conventional methods on previously dried hydrogen montmorillonite are unreliable owing to a decreased rate of exchange with inorganic cations. Our studies have led us to conclude that in all cases hydrogen montmorillonite was his final product, and therefore the reported decreases 